\title{
TRANSLATIONAL ADDITION THEOREMS FOR SPHERICAL LAPLACIAN FUNCTIONS AND THEIR APPLICATION TO BOUNDARY-VALUE PROBLEMS
}

\author{
BY \\ IOAN R. CIRIC (Department of Electrical and Computer Engineering, The University of Manitoba, \\ Canada) \\ AND
}

KUMARA S. C. M. KOTUWAGE (Department of Electrical and Computer Engineering, The University of Manitoba, Canada)

\begin{abstract}
General translational addition theorems are presented for spherical scalar Laplacian functions, and their application to boundary value problems is illustrated. By these theorems, the eigenfunction solutions in a system of spherical coordinates are expressed in terms of the spherical coordinates in another system, translated with respect to the first one. This allows for a rigorous analytic solution to be obtained for Laplacian and Poissonian fields in the presence of arbitrary configurations of spheres by imposing the exact boundary conditions. Complete formulations and solutions are presented for systems of electrically charged spheres and for arrays of perfect conductor spheres in external electric and magnetic fields. Illustrative computation examples are given for three-sphere systems. Numerical results of specified accuracy are generated, which are useful for validating various approximate numerical methods.
\end{abstract}

1. Introduction. Addition theorems for spherical functions are needed in order to obtain scalar or vector field problem solutions of controllable accuracy relative to multiple-sphere systems. To impose the exact boundary conditions at the surface of each sphere, the fields due to the presence of the other spheres, which can easily be expressed in terms of coordinates attached to those spheres, have to be 'translated' to the system of coordinates attached to the sphere at whose surface the boundary condition is to be imposed. Such theorems for spherical scalar wave functions were derived in Friedman and Russek, 1954, and for spherical vector wave functions in Stein, 1961, and Cruzan, 1962. The latter were used to analyze the electromagnetic wave scattering by systems of

Received August 1, 2012.

2010 Mathematics Subject Classification. Primary 35A99, 35A09, 65N99.

E-mail address: Ioan.Ciric@ad.umanitoba.ca

E-mail address: mksckumara@gmail.com 
conducting and dielectric spheres (Hamid, Ciric and Hamid (1990)). The general expressions of the addition theorems for the special case of spherical Laplacian functions are not available in the literature. In this paper, we obtain the general form of the translational addition theorems for scalar spherical Laplacian functions and illustrate their application to the solution of electric and magnetic field problems.

\section{Translational addition theorems for spherical scalar Laplacian functions.}

The addition theorems for scalar wave functions given in Cruzan, 1962, for the translation from the system of spherical coordinates $r, \theta, \phi$ to the system $r^{\prime}, \theta^{\prime}, \phi^{\prime}$ shown in Figure 1 is, for $r^{\prime} \leq r_{0}$,

$$
\begin{gathered}
z_{n}(k r) P_{n}^{m}(\cos \theta) \exp (i m \phi)=\sum_{\nu=o}^{\infty} \sum_{\mu=-\nu}^{\nu} \sum_{p}(-1)^{\mu} i^{\nu-n+p}(2 \nu+1) a_{\nu \mu p}^{n m} \\
\cdot j_{\nu}\left(k r^{\prime}\right) z_{p}\left(k r_{0}\right) P_{\nu}^{\mu}\left(\cos \theta^{\prime}\right) P_{p}^{m-\mu}\left(\cos \theta_{0}\right) \exp \left(i \mu \phi^{\prime}\right) \exp \left[i(m-\mu) \phi_{0}\right],
\end{gathered}
$$

where $i \equiv \sqrt{-1}, k$ is the wave number, $n$ and $m$ are integers, $n \geq 0,|m| \leq n, z_{n}$ stands for any of the spherical Bessel function $j_{n}$, Neumann function $n_{n}$, or Hankel functions, $P_{n}^{m}$ is the associated Legendre function of the first kind of degree $n$ and order $m$ (Smythe (1989)), and $a_{v \mu p}^{n m}$ are expansion coefficients defined in the Appendix. The sum over $p$ is over all of the values

$$
p=n+\nu, n+\nu-2, n+\nu-4, \ldots,|n-\nu|,
$$

for which the coefficient $a_{\nu \mu p}^{n m}$ is different from zero. For $r^{\prime} \geq r_{0}$, the addition theorem is obtained from (2.1) by interchanging $r^{\prime}, \theta^{\prime}, \phi^{\prime}$ and $r_{0}, \theta_{0}, \phi_{0}$, respectively.

The general form of the solution of the scalar Laplace equation in spherical coordinates $r, \theta, \phi$, for regions with $0 \leq \phi \leq 2 \pi$ and which contain sections of the negative $z$-axis, can be constructed in terms of two elementary solutions, i.e.,

$$
f_{n m}^{(1)}(r, \theta, \phi)=r^{n} P_{n}^{m}(\cos \theta) \exp (i m \phi), f_{n m}^{(2)}(r, \theta, \phi)=r^{-(n+1)} P_{n}^{m}(\cos \theta) \exp (i m \phi) .
$$

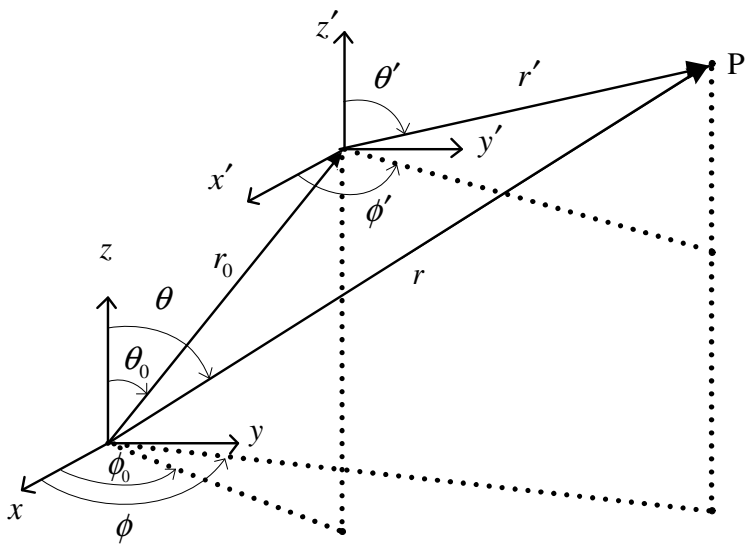

FIG. 1. Translation of the coordinate system 
To express $f_{n m}^{(1)}$ and $f_{n m}^{(2)}$ in the coordinates $r^{\prime}, \theta^{\prime}, \phi^{\prime}$ in a translated system of coordinates, we notice that the elementary solutions in (2.3) are simply related to $z_{n}(k r) P_{n}^{m}(\cos \theta) \exp (i m \phi)$. Indeed, taking $z_{n}$ to be $j_{n}$ and using the limiting value as $k \rightarrow 0$ (Abramovitz and Steigun (1965)),

$$
(k r)^{-n} j_{n}(k r) \rightarrow 1 /(2 n+1) ! !
$$

where ( )!! denotes the double factorial, (2.1) yields

$$
\begin{aligned}
& r^{n} P_{n}^{m}(\cos \theta) \exp (i m \phi)=\lim _{k \rightarrow 0}(2 n+1) ! ! \sum_{\nu=o}^{\infty} \sum_{\mu=-\nu}^{\nu} \sum_{p}(-1)^{\mu} i^{\nu-n+p} a_{\nu \mu p}^{n m} \\
& \cdot \frac{r^{\prime \nu} r_{0}^{p} k^{\nu-n+p}}{(2 \nu-1) ! !(2 p+1) ! !} P_{\nu}^{\mu}\left(\cos \theta^{\prime}\right) P_{p}^{m-\mu}\left(\cos \theta_{0}\right) \exp \left(i \mu \phi^{\prime}\right) \exp \left[i(m-\mu) \phi_{0}\right], r^{\prime} \leq r_{0} .
\end{aligned}
$$

Since $p \geq|n-\nu| \geq 0$ (see (2.2)), we have $\nu-n+p \geq 0$, and the only contributions to the summations are from the terms with the power of $k$ equal to zero, $\nu-n+p=0$, i.e., $p=n-\nu \geq 0$ and $\nu \leq n$. Using the expression of $a_{\nu \mu, n-\nu}^{n m}$ (see Appendix, (A.7)), (2.5) becomes

$$
\begin{gathered}
r^{n} P_{n}^{m}(\cos \theta) \exp (i m \phi)=(n+m) ! r_{0}^{n} \sum_{\nu=0}^{n} \sum_{\mu=-\nu}^{\nu}\left(\frac{r^{\prime}}{r_{0}}\right)^{\nu} \frac{1}{(\nu+\mu) !(n+m-\nu-\mu) !} \\
\cdot P_{\nu}^{\mu}\left(\cos \theta^{\prime}\right) P_{n-\nu}^{m-\mu}\left(\cos \theta_{0}\right) \exp \left(i \mu \phi^{\prime}\right) \exp \left[i(m-\mu) \phi_{0}\right], r^{\prime} \leq r_{0} .
\end{gathered}
$$

The translational theorem for $r^{\prime} \geq r_{0}$ can be obtained directly from (2.6) by interchanging $r^{\prime}, \theta^{\prime}, \phi^{\prime}$ and $r_{0}, \theta_{0}, \phi_{0}$, respectively. It should be remarked that the series in these two theorems have a finite number of terms and that each of the two expressions, for $r^{\prime} \leq r_{0}$ or $r^{\prime} \geq r_{0}$, can be employed for either $r^{\prime} \leq r_{0}$ or $r^{\prime} \geq r_{0}$ without restriction on the relative size of $r^{\prime}$ and $r_{0}$ (Stein (1961)).

Taking now $z_{n}$ to be $n_{n}$ and using the limiting value as $k \rightarrow 0$ (Abramovitz and Steigun (1965)),

$$
(k r)^{n+1} n_{n}(k r) \rightarrow-(2 n-1) ! !,
$$

substitution in (2.1) yields

$$
\begin{aligned}
& r^{-(n+1)} P_{n}^{m}(\cos \theta) \exp (i m \phi)=\lim _{k \rightarrow 0} \frac{1}{(2 n-1) ! !} \sum_{\nu=0}^{\infty} \sum_{\mu=-\nu}^{\nu} \sum_{p}(-1)^{\mu} i^{\nu-n+p} a_{\nu \mu p}^{n m} r^{\prime \nu} \\
& \cdot r_{0}^{-(p+1)} k^{\nu+n-p} \frac{(2 p-1) ! !}{(2 \nu-1) ! !} P_{\nu}^{\mu}\left(\cos \theta^{\prime}\right) P_{p}^{m-\mu}\left(\cos \theta_{0}\right) \exp \left(i \mu \phi^{\prime}\right) \exp \left[i(m-\mu) \phi_{0}\right], r^{\prime} \leq r_{0} .
\end{aligned}
$$

Since $p \leq n+\nu$ (see (2.2)), in the limit, only the terms with $p=n+\nu$ (when the power of $k$ is equal to zero) contribute to the summations. With the expression of $a_{\nu \mu, n+\nu}^{n m}$ in the Appendix (see (A.10)), (2.8) becomes

$$
\begin{aligned}
& r^{-(n+1)} P_{n}^{m}(\cos \theta) \exp (i m \phi)=\frac{r_{0}^{-(n+1)}}{(n-m) !} \sum_{\nu=0}^{\infty} \sum_{\mu=-\nu}^{\nu}(-1)^{\nu+\mu}\left(\frac{r^{\prime}}{r_{0}}\right)^{\nu} \frac{(n-m+\nu+\mu) !}{(\nu+\mu) !} \\
& \cdot P_{\nu}^{\mu}\left(\cos \theta^{\prime}\right) P_{n+\nu}^{m-\mu}\left(\cos \theta_{0}\right) \exp \left(i \mu \phi^{\prime}\right) \exp \left[i(m-\mu) \phi_{0}\right], r^{\prime} \leq r_{0} .
\end{aligned}
$$


The translational addition theorem for $r^{\prime} \geq r_{0}$ is obtained in a similar manner or directly from (2.9) by interchanging $r^{\prime}, \theta^{\prime}, \phi^{\prime}$ and $r_{0}, \theta_{0}, \phi_{0}$, respectively.

In the special case when the translation is along the $z$-axis, $\phi^{\prime}=\phi, \theta_{0}=0$ or $\pi$, $\left(r_{0}=z_{0}\right)$, and (2.6) and (2.9) reduce, respectively, to

$$
\begin{aligned}
& r^{n} P_{n}^{m}(\cos \theta)=(n+m) ! r_{0}^{n} \sum_{\nu=|m|}^{n}\left(\frac{r^{\prime}}{r_{0}}\right)^{\nu} \frac{1}{(n-\nu) !(m+\nu) !} P_{\nu}^{m}\left(\cos \theta^{\prime}\right) P_{n-\nu}\left(\cos \theta_{0}\right), r^{\prime} \leq r_{0}, \\
& r^{-(n+1)} P_{n}^{m}(\cos \theta)=\frac{r_{0}^{-(n+1)}}{(n-m) !} \sum_{\nu=|m|}^{\infty}(-1)^{m+\nu}\left(\frac{r^{\prime}}{r_{0}}\right)^{\nu} \frac{(n+v) !}{(m+\nu) !} P_{\nu}^{m}\left(\cos \theta^{\prime}\right) P_{n+\nu}\left(\cos \theta_{0}\right),
\end{aligned}
$$

3. Application to Laplacian scalar potential problems. Consider a system of $S$ spheres arbitrarily located, the spheres $p$ and $q$ being shown in Figure 2. The Cartesian axes of all the spheres are, respectively, parallel. The center of the sphere $q$ has the spherical coordinates $r_{p q}, \theta_{p q}, \phi_{p q}$ in the system of coordinates attached to the sphere $p$, and the spherical coordinates of the center of $p$ in the system attached to $q$ are $r_{q p}=r_{p q}$, $\theta_{q p}=\pi-\theta_{p q}, \phi_{q p}=\pi+\phi_{p q}$.

The scalar potential due to the presence of the sphere $p$ can be written as (see (2.3))

$$
\Phi_{p}\left(r_{p}, \theta_{p}, \phi_{p}\right)=\sum_{n=0}^{\infty} \sum_{m=-n}^{n} A_{n m}^{(p)} r_{p}^{-(n+1)} P_{n}^{m}\left(\cos \theta_{p}\right) \exp \left(i m \phi_{p}\right), r_{p} \geq a_{p}, p=1,2, \ldots, S,
$$

where $r_{p}, \theta_{p}, \phi_{p}$ are the spherical coordinates of the observation point, of position vector $\mathbf{r} \equiv \mathbf{r}_{\mathbf{p}}$, in the system $p$ and $A_{n m}^{(p)}$ are constants of integration to be determined. The resultant potential at any point outside the spheres due to the presence of all of them and of an external applied field is

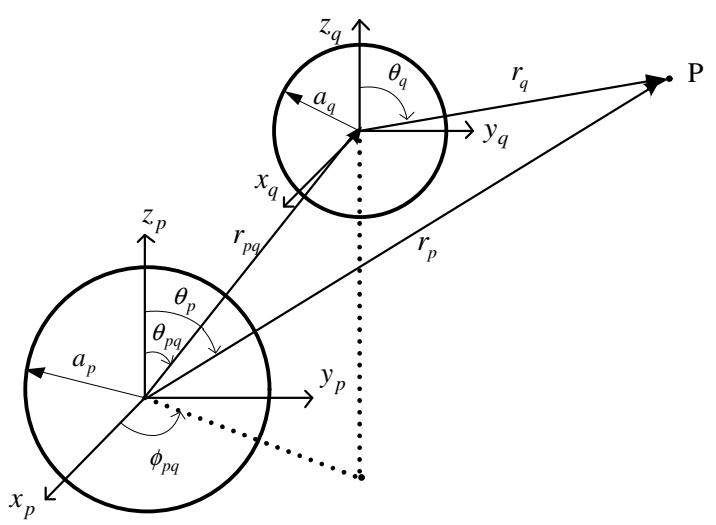

Fig. 2. Pair of spheres $p$ and $q$ 


$$
\Phi(\mathbf{r})=\sum_{p=1}^{S} \Phi_{p}\left(r_{p}, \theta_{p}, \phi_{p}\right)+\Phi_{0}(\mathbf{r})
$$

where $\Phi_{0}$ is the potential due to the applied field.

When the material inside a sphere $p$ is homogeneous and field-penetrable, then the potential here is

$$
\Phi_{i n}^{(p)}\left(r_{p}, \theta_{p}, \phi_{p}\right)=\sum_{n=0}^{\infty} \sum_{m=-n}^{n} B_{n m}^{(p)} r_{p}^{n} P_{n}^{m}\left(\cos \theta_{p}\right) \exp \left(i m \phi_{p}\right), r_{p} \leq a_{p}
$$

with the constants $B_{n m}^{(p)}$ to be determined.

In order to impose the boundary conditions at the surface of the sphere $p$, the potential in (3.2) is transformed to

$$
\begin{array}{r}
\Phi^{(p)}\left(r_{p}, \theta_{p}, \phi_{p}\right)=\Phi_{p}\left(r_{p}, \theta_{p}, \phi_{p}\right)+\sum_{\substack{q=1 \\
q \neq p}}^{S} \Phi_{q}^{(p)}\left(r_{p}, \theta_{p}, \phi_{p}\right)+\Phi_{0}^{(p)}\left(r_{p}, \theta_{p}, \phi_{p}\right), \\
r_{p} \geq a_{p}, \\
p=1,2, \ldots, S,
\end{array}
$$

where $\Phi_{0}^{(p)}$ is $\Phi_{0}$ expressed in coordinates $r_{p}, \theta_{p}, \phi_{p}$ and $\Phi_{q}^{(p)}$ is the potential $\Phi_{q}\left(r_{q}, \theta_{q}, \phi_{q}\right)$ (see (3.1)) in the system of coordinates $r_{p}, \theta_{p}, \phi_{p}$ obtained by translation from the system of coordinates $r_{q}, \theta_{q}, \phi_{q}$, i.e. (see (2.9)),

$$
\begin{gathered}
\Phi_{q}^{(p)}\left(r_{p}, \theta_{p}, \phi_{p}\right)=\sum_{n=0}^{\infty} \sum_{m=-n}^{n} A_{n m}^{(q)} \frac{r_{q p}^{-(n+1)}}{(n-m) !} \sum_{\nu=0}^{\infty} \sum_{\mu=-\nu}^{\nu}(-1)^{\nu+\mu}\left(\frac{r_{p}}{r_{q p}}\right)^{\nu} \frac{(n-m+v+\mu) !}{(v+\mu) !} \\
\cdot P_{\nu}^{\mu}\left(\cos \theta_{p}\right) P_{n+\nu}^{m-\mu}\left(\cos \theta_{q p}\right) \exp \left(i \mu \phi_{p}\right) \exp \left[i(m-\mu) \phi_{q p}\right], r_{p} \leq r_{q p} .
\end{gathered}
$$

The unknown constants of integration $A_{n m}^{(p)}, B_{n m}^{(p)}, p=1,2, \ldots, S$, are determined by imposing the boundary conditions at the surfaces $r_{p}=a_{p}$ of all the spheres. When a surface $r_{p}=a_{p}$ is equipotential, for instance, then the (Dirichlet) boundary condition is (with (3.4) and (3.5)) $\Phi^{(p)}\left(a_{p}, \theta_{p}, \phi_{p}\right)=V_{p}$, where $V_{p}$ is the given potential of the sphere $p$. When Neumann boundary conditions are to be imposed at a surface $r_{p}=a_{p}$, then one needs the normal derivative $\partial \Phi^{(p)} / \partial r_{p}$ at $r_{p}=a_{p}$ calculated from (3.4) and (3.5).

In the case of a system of spheres with a common $z$-axis and axisymmetric boundary conditions and applied external field, there is no dependence on the variable $\phi$ and, thus, $m=0$ in (3.1), (3.3), and $m=0, \mu=0$ in (3.5) (see examples in sections 4.2 and 4.3 below).

4. Illustrative examples. In what follows, the theorems in (2.9) and (2.11) are applied to calculate the electrostatic field of systems of metallic spheres electrically charged or immersed in external fields, as well as the magnetic field in the presence of configurations of magnetic spheres. 


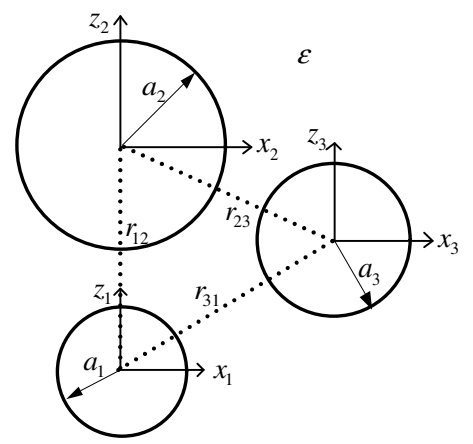

FIG. 3. System of spheres in arbitrary configuration

4.1. Electrically charged metallic spheres in arbitrary configurations. Consider for illustration a system of $S$ conducting spheres at given electric potentials $V_{p}$ (as shown in Figure 3 for $S=3$ ), with the medium outside the spheres being homogeneous, of permittivity $\varepsilon$. The electrostatic potential is given by (3.1), (3.2), with $\Phi_{0} \equiv 0$. The boundary conditions at the surfaces of the $S$ spheres are (see (3.4))

$$
\Phi_{p}\left(a_{p}, \theta_{p}, \phi_{p}\right)+\sum_{\substack{q=1 \\ q \neq p}}^{S} \Phi_{q}^{(p)}\left(a_{p}, \theta_{p}, \phi_{p}\right)=V_{p}, p=1,2, \ldots, S .
$$

Multiplying both sides of the equations (4.1) with $P_{n}^{m}\left(\cos \theta_{p}\right) \sin \theta_{p} \exp \left(-i m \phi_{p}\right)$ and applying the orthogonality properties of the spherical harmonics (Smythe (1989)) yields an infinite system of linear algebraic equations for the unknown constants of integration $A_{n m}^{(p)}, p=1,2, \ldots, S$, which can be written in the form

$$
\begin{gathered}
A_{n^{\prime} m^{\prime}}^{(p)}+(-1)^{n^{\prime}+m^{\prime}} a_{p}^{n^{\prime}+1} \frac{1}{\left(n^{\prime}+m^{\prime}\right) !} \sum_{\substack{q=1 \\
q \neq p}}^{S} \sum_{n=0}^{\infty} \sum_{m=-n}^{n} A_{n m}^{(q)} r_{q p}^{-(n+1)}\left(\frac{a_{p}}{r_{q p}}\right)^{n^{\prime}} \\
. \frac{\left(n-m+n^{\prime}+m^{\prime}\right) !}{(n-m) !} P_{n+n^{\prime}}^{m-m^{\prime}}\left(\cos \theta_{q p}\right) \exp \left[i\left(m-m^{\prime}\right) \phi_{q p}\right]=V_{p} a_{p} \delta_{n^{\prime} 0}, \\
p=1,2, \ldots, S ; n^{\prime}=0,1,2, \ldots ; m^{\prime}=-n^{\prime},-n^{\prime}+1, \ldots, n^{\prime},
\end{gathered}
$$

where $\delta_{n^{\prime} 0}$ is the Kronecker symbol.

The total charge of the sphere $p$ is obtained as

$$
Q_{p}=4 \pi \varepsilon A_{00}^{(p)}, p=1,2, \ldots, S .
$$

Numerical results are generated by truncating appropriately the infinite system (4.2). To give a concrete example for the system of three spheres $(S=3)$ in Figure 3, with $a_{1}=3 \mathrm{~cm}, a_{2}=5 \mathrm{~cm}, a_{3}=4 \mathrm{~cm}$ and $r_{12}=r_{23}=r_{31}=10 \mathrm{~cm}$, a 5-digit accuracy was achieved for the charge values when the infinite series were truncated to $n=10$. For a free space outside the spheres, the following values in $\mathrm{pF}$ have been obtained for the capacitance coefficients (Smythe (1989)): 
$c_{11}=4.4710, c_{22}=8.4680, c_{33}=6.6793, c_{12}=c_{21}=-1.7474, c_{23}=c_{32}=-3.0661$, $c_{31}=c_{13}=-1.0902$.

4.2. Electric field intensification in the presence of coaxial conducting spheres. Consider now an array of $S$ coaxial metallic spheres in a uniform external electric field of intensity $\mathbf{E}_{\mathbf{0}}$ oriented along the common $z$-axis of the spheres. Each of the spheres has a total electric charge equal to zero, and the medium outside the spheres is homogeneous.

There is no dependence on the variable $\phi$, and $\theta_{q p}$ is either 0 or $\pi$; the potential in (3.4) becomes (with $m=0, \mu=0$ in (3.1) and (3.5))

$$
\begin{gathered}
\Phi^{(p)}\left(r_{p}, \theta_{p}\right)=\sum_{n=0}^{\infty} A_{n}^{(p)} r_{p}^{-(n+1)} P_{n}\left(\cos \theta_{p}\right)+\sum_{\substack{q=1 \\
q \neq p}}^{S} \sum_{n=0}^{\infty} A_{n}^{(q)} \frac{r_{q p}^{-(n+1)}}{n !} \sum_{\nu=0}^{\infty}(-1)^{\nu}\left(\frac{r_{p}}{r_{q p}}\right)^{\nu} \\
\cdot \frac{(n+\nu) !}{\nu !} P_{\nu}\left(\cos \theta_{p}\right) P_{n+\nu}\left(\cos \theta_{q p}\right)-E_{0} z+C_{p}, r_{p} \geq a_{p}, p=1,2, \ldots, S,
\end{gathered}
$$

where $-E_{0} z$ is the potential corresponding to $\mathbf{E}_{\mathbf{0}}$ with the origin of the $z$-axis chosen arbitrarily, for instance at the center of one of the spheres, the additive constants $C_{p}$ being fixed in terms of the reference point for the potential of the external field. From the condition $Q_{p}=0, p=1,2, \ldots, S, S$ constants of integration are determined, namely (see $(4.3)) A_{0}^{(p)}=0, p=1,2, \ldots, S$.

The spheres are equipotential, i.e., $\Phi^{(p)}\left(a_{p}, \theta_{p}\right)=V_{p}, p=1,2, \ldots, S$, but the potentials $V_{p}$ are now unknown. With $E_{0} z=E_{0} r_{p} \cos \theta_{p} \equiv E_{0} r_{p} P_{1}\left(\cos \theta_{p}\right)$ in (4.4) and by using the orthogonality properties of the Legendre polynomials (Smythe (1989)), we get an infinite system of linear equations for the unknowns $\left(V_{p}-C_{p}\right)$ and $A_{n}^{(p)}, p=1,2, \ldots, S$, $n=1,2,3, \ldots$, in the form

$$
\begin{aligned}
& \left(V_{p}-C_{p}\right)-\sum_{\substack{q=1 \\
q \neq p}}^{S} \sum_{n=1}^{\infty} A_{n}^{(q)} r_{q p}^{-(n+1)} P_{n}\left(\cos \theta_{q p}\right)=0, \\
& A_{n^{\prime}}^{(p)}+(-1)^{n^{\prime}} a_{p}^{n^{\prime}+1} \frac{1}{n^{\prime} !} \sum_{\substack{q=1 \\
q \neq p}}^{S} \sum_{n=1}^{\infty} A_{n}^{(q)} r_{q p}^{-(n+1)}\left(\frac{a_{p}}{r_{q p}}\right)^{n^{\prime}} \frac{\left(n+n^{\prime}\right) !}{n !} P_{n+n^{\prime}}\left(\cos \theta_{q p}\right)=\frac{2}{3} a_{p}^{3} E_{0} \delta_{n^{\prime} 1}, \\
& \quad p=1,2, \ldots, \mathrm{S} ; n^{\prime}=1,2,3, \ldots
\end{aligned}
$$

The electric field intensity at points on the sphere $p$ is evaluated as $E\left(\theta_{p}\right)=$ $-\partial \Phi^{(p)} / \partial r_{p}$ at $r_{p}=a_{p}$. Numerical results are presented in Table 1 a) for a system of three identical metallic spheres as shown in Figure 4. To generate results of a specified accuracy, the infinite series in (4.5) are truncated to a finite number of terms, $n=n^{\prime}=N$, which increases when the relative value of the gap $g$ decreases. At the points $\mathrm{A}, \mathrm{B}$ and $\mathrm{C}$ in Figure 4, results of 5-digit accuracy are obtained with $N=8$ for $g / a \geq 1, N=40$ for $g / a=0.1, N=120$ for $g / a=0.01$ and $N=170$ for $g / a=0.005$. A 3-digit accuracy is obtained with $N=30,75$ and 100 , respectively, for $g / a=0.1,0.01$ and 0.005 . One notices the strong intensification of the electric field in the gap regions of the spheres when they are very close to each other. 


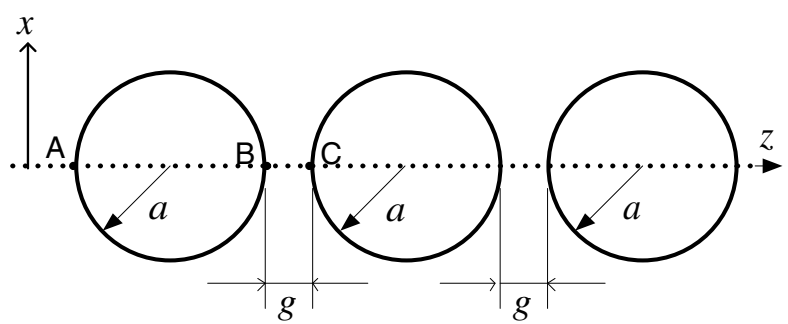

FIG. 4. Three coaxial spheres

TABLE 1. Field intensification at the points A, B and C in Figure 4 for three identical spheres in a uniform field:

a) conducting spheres in an axial electric field, $\mathbf{E}_{\mathbf{0}}=E_{0} \hat{\mathbf{z}}$

\begin{tabular}{|c|c|c|c|c|c|c|c|c|c|c|}
\hline Point & $\begin{array}{c}\boldsymbol{g} / \boldsymbol{a} \\
\text { Field }\end{array}$ & $\mathbf{5 . 0 0}$ & $\mathbf{1 . 0 0}$ & $\mathbf{0 . 5 0}$ & $\mathbf{0 . 2 0}$ & $\mathbf{0 . 1 0}$ & $\mathbf{0 . 0 5}$ & $\mathbf{0 . 0 2}$ & $\mathbf{0 . 0 1}$ & $\mathbf{0 . 0 0 5}$ \\
\hline $\mathrm{A}$ & $E_{z} / E_{0}$ & 3.01460 & 3.15137 & 3.27008 & 3.45386 & 3.59462 & 3.72704 & 3.88319 & 3.98623 & 4.07715 \\
\hline $\mathrm{B}$ & $E_{z} / E_{0}$ & 3.02885 & 3.80677 & 5.22587 & 9.49097 & 16.1108 & 28.3915 & 62.0759 & 113.922 & 210.911 \\
\hline $\mathrm{C}$ & $E_{z} / E_{0}$ & 3.03875 & 3.85497 & 5.25679 & 9.49459 & 16.1109 & 28.3915 & 62.0759 & 113.922 & 210.911 \\
\hline
\end{tabular}

b) perfect conductor spheres in a transverse magnetic field, $\mathbf{H}_{\mathbf{0}}=H_{0} \hat{\mathbf{x}}$

\begin{tabular}{|c|c|c|c|c|c|c|c|c|c|c|}
\hline Point & $\begin{array}{c}\text { F } / \boldsymbol{a} \\
\text { Field }\end{array}$ & $\mathbf{5 . 0 0}$ & $\mathbf{1 . 0 0}$ & $\mathbf{0 . 5 0}$ & $\mathbf{0 . 2 0}$ & $\mathbf{0 . 1 0}$ & $\mathbf{0 . 0 5}$ & $\mathbf{0 . 0 2}$ & $\mathbf{0 . 0 1}$ & $\mathbf{0 . 0 0 5}$ \\
\hline $\mathrm{A}$ & $H_{x} / H_{0}$ & 1.50161 & 1.51295 & 1.51971 & 1.52663 & 1.52990 & 1.53186 & 1.53320 & 1.53369 & 1.53395 \\
\hline $\mathrm{B}$ & $H_{x} / H_{0}$ & 1.50399 & 1.61580 & 1.79735 & 2.21176 & 2.65806 & 3.22789 & 4.20205 & 5.14121 & 6.29495 \\
\hline $\mathrm{C}$ & $H_{x} / H_{0}$ & 1.50504 & 1.61936 & 1.79923 & 2.21193 & 2.65807 & 3.22789 & 4.20205 & 5.14121 & 6.29495 \\
\hline
\end{tabular}

4.3. Arrays of coaxial perfect conductor spheres in transverse magnetic fields. For a system of $S$ perfect conductor spheres, the Laplacian scalar magnetic potential satisfies the general equations (3.1)-(3.5) with a zero boundary value for the Neumann condition at the surface of each sphere, i.e., $\partial \Phi^{(p)} /\left.\partial r_{p}\right|_{r_{p}=a_{p}}=0, p=1,2, \ldots, S$. When the spheres are coaxial and in the presence of a uniform transverse magnetic field $\mathbf{H}_{\mathbf{0}}=H_{0} \hat{\mathbf{x}}$ (see Figure 4), the resultant potential in spherical coordinates attached to the sphere $p$ has the form (see (3.4), (3.5) and (2.11))

$$
\begin{array}{r}
\Phi^{(p)}\left(r_{p}, \theta_{p}, \phi_{p}\right)=\sum_{n=1}^{\infty} A_{n}^{(p)} r_{p}^{-(n+1)} P_{n}^{1}\left(\cos \theta_{p}\right) \cos \phi_{p}+\sum_{\substack{q=1 \\
q \neq p}}^{S} \sum_{n=1}^{\infty} A_{n}^{(q)} \frac{r_{q p}^{-(n+1)}}{(n-1) !} \sum_{\nu=1}^{\infty}(-1)^{\nu+1} \\
\cdot\left(\frac{r_{p}}{r_{q p}}\right)^{\nu} \frac{(n+\nu) !}{(\nu+1) !} P_{n+\nu}\left(\cos \theta_{q p}\right) P_{v}^{1}\left(\cos \theta_{p}\right) \cos \phi_{p}-H_{0} r_{p} \sin \theta_{p} \cos \phi_{p}, r_{p} \geq a_{p}, \\
p=1,2, \ldots, S,
\end{array}
$$


with $\theta_{q p}=0$ or $\pi$. The following infinite system of equations is derived for the unknowns $A_{n}^{(p)}, p=1,2, \ldots, S, n=1,2,3, \ldots$, :

$$
\begin{aligned}
A_{n^{\prime}}^{(p)}+(-1)^{n^{\prime}} a_{p}^{n^{\prime}+1} \frac{n^{\prime}}{\left(n^{\prime}+1\right)\left(n^{\prime}+1\right) !} & \sum_{\substack{q=1 \\
q \neq p}}^{S} \sum_{n=1}^{\infty} A_{n}^{(q)} r_{q p}^{-(n+1)}\left(\frac{a_{p}}{r_{q p}}\right)^{n^{\prime}} \frac{\left(n+n^{\prime}\right) !}{(n-1) !} P_{n+n^{\prime}}\left(\cos \theta_{q p}\right) \\
& =-\frac{1}{2} a_{p}^{3} H_{0} \delta_{n^{\prime} 1}, p=1,2, \ldots, \mathrm{S} ; n^{\prime}=1,2,3, \ldots
\end{aligned}
$$

In Table $1 \mathrm{~b}$ ), we present numerical results for a system of three coaxial identical perfect conductor spheres shown in Figure 4. Again, the number $n=n^{\prime}=N$ to be retained in the infinite series in (4.7) in order to obtain a desired accuracy of the numerical results increases as the relative gap, $g / a$, decreases. For the magnetic field intensity at the points $\mathrm{A}, \mathrm{B}$ and $\mathrm{C}$ in Figure 4, a 5-digit accuracy is obtained with $N=7$ for $g / a \geq 1, N=35$ for $g / a=0.1, N=125$ for $g / a=0.01$, and $N=175$ for $g / a=0.005$, while for a 3-digit accuracy one needs $N=20,100$ and 120, respectively, for $g / a=0.1,0.01$ and 0.005 . An appreciable intensification of the magnetic field at the points $\mathrm{B}$ and $\mathrm{C}$ is observed for small gaps between the spheres though much less strong than the intensification of the electric field at these points in the example considered in Table 1 a).

5. Remarks and conclusion. The general expressions in (2.6) and (2.9) for $r^{\prime} \leq r_{0}$, as well as the corresponding expressions for $r^{\prime} \geq r_{0}$, take a simpler form in various particular situations. In the cases, for instance, when the centers of the spheres are on the same plane (the configuration with $S=3$ in Figure 3, considered in section 4.1, is included in this category), then this plane is chosen to be the $z x$-plane such that $\phi_{q p}=0, p, q=1,2, \ldots, S$, and, as a consequence, all the entries and the unknowns (the constants of integration $A_{n m}^{(p)}$ ) in the system of equations (4.2) are real numbers. When all the spheres have a common $z$-axis (as in sections 4.2 and 4.3), the translational addition theorems only contain single series (see (2.10) and (2.11)) which yield much smaller truncated systems of equations (compare (4.5) with (4.2)) for an imposed accuracy of the numerical results. Comparing the classical expression in terms of Legendre polynomials of the inverse distance between two arbitrary points on a unit sphere with that obtained as a special case for $n=0, r^{\prime}=r_{0}=1$ of our formula in (2.9) yields directly the celebrated Legendre's addition theorem (Hobson (1965)). A variety of simplified expressions for positive and negative powers of the distance between two points located at particular positions can be generated from (2.6), (2.9) and from their companions for $r^{\prime} \geq r_{0}$.

The formulations presented in this paper particularized for a system of two spheres constitute an alternative to the classical formulations using the method of images in the case of equipotential spheres or employing the bispherical coordinates (Morse and Feshbach (1953), Ciric and Kotuwage (2011)). The application of the translational addition theorems to the field problems in section 4 can be performed in the same way for other scalar Laplacian fields relative to multiple-sphere systems, for example for temperature distribution and in fluid mechanics, thus extending the class of problems which can be 
solved by exact analytic methods. This includes problems relative to spheres with nonhomogeneous boundary conditions and also field-penetrable spheres. As well, the results in this paper are relevant to studies regarding the behavior of cloud water droplets in external electric fields, the determination of electric and magnetic field intensification, and of forces on particles in colloidal suspensions, the response of nanostructures to electromagnetic fields, where, at a first approximation, the intervening small bodies or particles are modeled as spheres. The formulation and solution for perfect conductor spheres in the presence of magnetic fields can also be used for calculating the field intensity, induced electric currents, losses and forces in problems of pronounced skin effect for solid conductors at sufficiently high frequencies, when the field depth of penetration is negligible with respect to the local radii of curvature of the bodies involved.

Appendix A. Expansion coefficients $a_{\nu \mu, n \mp \nu}^{n m}$ in (2.5) and (2.8). The general expression of the expansion coefficients $a_{\nu \mu p}^{n m}$ in (2.1) is (Cruzan (1962))

$$
\begin{aligned}
a_{\nu \mu p}^{n m}=(-1)^{m-\mu}(2 p+1)\left[\frac{(n+m) !(\nu-\mu) !(p-m+\mu) !}{(n-m) !(\nu+\mu) !(p+m-\mu) !}\right]^{1 / 2} & {\left[\begin{array}{ccc}
n & \nu & p \\
0 & 0 & 0
\end{array}\right] } \\
& \cdot\left[\begin{array}{ccc}
n & \nu & p \\
m & -\mu & -m+\mu
\end{array}\right],
\end{aligned}
$$

where the Wigner "3j" symbol is calculated using the formula (Messiah (1999))

$$
\begin{aligned}
& {\left[\begin{array}{lll}
a & b & c \\
\alpha & \beta & \gamma
\end{array}\right]=(-1)^{a-b-\gamma}[(a+\alpha) !(a-\alpha) !(b+\beta) !(b-\beta) !(c+\gamma) !(c-\gamma) ! \Delta(a, b, c)]^{1 / 2}} \\
& \cdot \sum_{s}(-1)^{s}[s !(a+b-c-s) !(a-\alpha-s) !(b+\beta-s) !(c-b+\alpha+s) !(c-a-\beta+s) !]^{-1}
\end{aligned}
$$

with

$$
\alpha+\beta+\gamma=0,|a-b| \leq c \leq a+b
$$

and

$$
\Delta(a, b, c) \equiv \frac{(a+b-c) !(b+c-a) !(c+a-b) !}{(a+b+c+1) !} .
$$

$\sum_{s}$ extends over all integral values of $s$ for which the arguments of the factorials are positive or null $(0 !=1)$.

In the special case (see (2.5)) when $p=n-\nu \geq 0$, we obtain

$$
\begin{aligned}
& {\left[\begin{array}{ccc}
n & \nu & n-\nu \\
m & -\mu & -m+\mu
\end{array}\right]} \\
& =(-1)^{n+m}\left[\frac{(n+m) !(n-m) !}{(\nu+\mu) !(\nu-\mu) !(n+m-\nu-\mu) !(n-m-\nu+\mu) !}\right]^{1 / 2}\left[\frac{(2 \nu) ![2(n-\nu)] !}{(2 n+1) !}\right]^{1 / 2},
\end{aligned}
$$

and, for $m=\mu=0$,

$$
\left[\begin{array}{ccc}
n & \nu & n-\nu \\
0 & 0 & 0
\end{array}\right]=(-1)^{n} \frac{n !}{\nu !(n-\nu) !}\left[\frac{(2 \nu) ![2(n-\nu)] !}{(2 n+1) !}\right]^{1 / 2}
$$


Thus,

$$
a_{\nu \mu, n-\nu}^{n m}=(-1)^{\mu} \frac{n !(n+m) !(2 \nu) ![2(n-\nu)+1] !}{(2 n+1) ! \nu !(\nu+\mu) !(n-\nu) !(n+m-\nu-\mu) !}, \quad|m-\mu| \leq n-\nu .
$$

In the case (see (2.8)) when $p=n+\nu$, we get

$$
\begin{aligned}
& {\left[\begin{array}{ccc}
n & \nu & n+\nu \\
m & -\mu & -m+\mu
\end{array}\right]=(-1)^{(n+m-\nu-\mu)} } {\left[\frac{(n+m+\nu-\mu) !(n-m+\nu+\mu) !}{(n+m) !(n-m) !(\nu+\mu) !(\nu-\mu) !}\right]^{1 / 2} } \\
& \cdot\left[\frac{(2 n) !(2 \nu) !}{[2(n+\nu)+1] !}\right]^{1 / 2}
\end{aligned}
$$

and, for $m=\mu=0$,

$$
\left[\begin{array}{ccc}
n & \nu & n+\nu \\
0 & 0 & 0
\end{array}\right]=(-1)^{n-v} \frac{(n+\nu) !}{n ! \nu !}\left[\frac{(2 n) !(2 \nu) !}{[2(n+\nu)+1] !}\right]^{1 / 2} .
$$

Thus,

$$
a_{\nu \mu, n+\nu}^{n m}=\frac{(2 n) !(2 \nu !)(n+\nu) !(n-m+\nu+\mu) !}{n !(n-m) ! \nu !(\nu+\mu) ![2(n+\nu)] !} .
$$

\section{REFERENCES}

[1] M. Abramovitz and I. A. Stegun, Handbook of Mathematical Functions, p. 437, Dover, 1965.

[2] I. R. Ciric and K. S. C. M. Kotuwage, Benchmark Solutions for Magnetic Fields in the Presence of Two Superconducting Spheres, Materials Science Forum, 721, pp. 21-26, 2012.

[3] Orval R. Cruzan, Translational addition theorems for spherical vector wave functions, Quart. Appl. Math. 20 (1962/1963), 33-40. MR0132851 (24 \#A2687)

[4] Bernard Friedman and Joy Russek, Addition theorems for spherical waves, Quart. Appl. Math. 12 (1954), 13-23. MR0060649 (15,702h)

[5] A. K. Hamid, I. R. Ciric and M. Hamid, Electromagnetic Scattering by an Arbitrary Configuration of Dielectric Spheres, Canadian Journal of Physics, 68(12), pp. 1419-1428, 1990.

[6] E. W. Hobson, The Theory of Spherical and Ellipsoidal Harmonics, pp. 141-144, Chelsea, 1965.

[7] A. Messiah, Quantum Mechanics, pp. 1054-1060, Mineola, Dover, 1999.

[8] Philip M. Morse and Herman Feshbach, Methods of theoretical physics. 2 volumes, McGraw-Hill Book Co., Inc., New York, 1953. MR0059774 (15,583h)

[9] W. R. Smythe, Static and Dynamic Electricity, 3rd ed., Chps. 2 and 5, McGraw-Hill, 1989.

[10] Seymour Stein, Addition theorems for spherical wave functions, Quart. Appl. Math. 19 (1961), 15-24. MR0120407(22 \#11161) 